\title{
Secuencia de producción lítica en el sitio Mesada del Agua Salada, Caspinchango, Valle de Yocavil
}

\author{
Juan Pablo CARBonelli \\ Museo Etnográfico (Buenos Aires) - CONICET \\ juanp.carbonelli@gmail.com
}

Recibido: 22 de diciembre de 2010 / 16 de julio de 2012

Aceptado: 19 de julio de 2012

\section{RESUMEN}

En este trabajo se analiza la secuencia de producción de la tecnología lítica en el sitio denominado Mesada del Agua Salada, situado en el Valle de Yocavil, Catamarca. Este sitio se halla conformado por dos recintos enclavados en la falda del Aconquija, uno de ellos que corresponde cronológicamente al primer milenio d.C., mientras que el segundo corresponde al Período Tardío. En ambos recintos se registraron en forma predominante evidencias de actividades de manufactura (talla y regularización de filos) y posiblemente uso. En menor medida se registraron evidencias de extracción y reactivación de instrumentos. En líneas generales, los instrumentos del sitio Mesada del Agua Salada registran un bajo grado de talla, siendo la obsidiana la materia prima que muestra un mayor esfuerzo tecnológico. Destacamos la continuidad en el consumo de las mismas variedades de obsidianas y la convergencia en las mismas opciones tecnológicas desde el Período Formativo hasta el Tardío en el sur del Valle de Yocavil.

Palabras clave: Período Formativo, Valle de Yocavil, secuencia de producción, tecnología lítica.

\section{Sequence of Lithic Production at the Site of Mesada del Agua Salada, Caspinchango, Yocavil Valley}

\begin{abstract}
In this paper we analyze the production sequence of lithic technology at the site called «Mesada del Agua Salada», located in the Yocavil Valley, Catamarca. This site is made up of two rooms nestled in the foothills of Aconquija, one of which corresponds chronologically to the first millennium $\mathrm{AD}$, while the second corresponds to the Later Period. Evidences of manufacturing activities (formatization and regularization of edges) and possibly use are predominantly recorded. Evidences of extraction and reactivation of instruments are identified in lesser extent. In general, the instruments of «Mesada del Agua Salada» show a low degree of formatization, being obsidian the raw material which demonstrates a greater technological effort. We emphasize the continuity in the use of the same varieties of obsidian and the convergence in the same technological options from the Formative Period to the Late Period in the Southern Yocavil Valley.
\end{abstract}

Key words: Formative Period, Yocavil Valley, sequence of production, lithic technology.

Sumario: 1. Introducción. 2. Procedencia y contexto de la muestra. 3. Material y metodología. 4. Resultados obtenidos. 5. Discusión y conclusiones. 6. Referencias bibliográficas.

\section{Introducción}

La tecnología lítica de los sitios agropastoriles en la zona de valles en momentos tempranos constituye una temática que recientemente ha ganado interés en los estudios del Noroeste Argentino (Carbonelli 2009a, 2011; Gaál y Carbonelli 2009; Lazzari 1998, 1999; Míguez et.al 2009; Scattolin et al. 2001; Somonte 2005, 2009); también 
en esta última década se ha acrecentado la investigación sobre la tecnología lítica en el período denominado Tardío (desde $c a$. 1000 AP) en la misma región (Álvarez 2004; Avalos 2003; Chaparro 2002, 2007, 2008-09, 2009; Elías 2007, 2010; Elías y Escola 2010; Flores y Wynveldt 2009; Gaál 2011; Ledesma 2003; Sprovieri 2007). Sin embargo, el número de sitios analizados, particularmente en el Valle de Yocavil, aún sigue siendo escaso. Por lo tanto, consideramos que el estudio de la tecnología lítica del sitio Mesada del Agua Salada, en la localidad de Caspinchango, contribuirá al conocimiento y caracterización de la interacción de los mencionados grupos humanos con los distintos recursos líticos.

La consolidación de un tipo de sociedad «formativa» se produce entre el 3000 y el 1000 AP (Tarragó 1999). Este tipo de sociedades se caracterizaron por organizarse en función de cierta opción productiva (agrícola y/o pastoril), complementada con caza y recolección (Olivera 1988). Esto obligaba a adoptar un determinado grado de sedentarismo y a la incorporación de cierta tecnología adecuada, por ejemplo la alfarería (Olivera 1988).

La intersección entre ambos conceptos, Formativo y tecnología lítica, en el curso de las investigaciones llevadas cabo hasta este momento en las regiones de valle, ha dado lugar a un corpus de conocimiento que permite establecer que: a) en la estrategia de aprovisionamiento predominan las rocas locales sobre las alóctonas, en particular de fuentes secundarias (Carbonelli 2009; Míguez et.al 2009; Oliszewski et.al 2010; Somonte 2005; 2009); b) las ocupaciones formativas interactúan y explotan de forma disímil cada materia prima (Carbonelli 2009a, 2010; Gaál y Carbonelli 2009; Lazzari 1998; Scattolin et al. 2001; Somonte 2009); c) se efectúan procesos de reciclaje y retomación de las materias primas, utilizando fuentes terciarias (Hocsman et al. 2003; Somonte 2005, 2009); d) se han identificado complejas redes de interacción y aprovisionamiento de materias primas alóctonas, en particular de la obsidiana (Escola y Hocsman 2007), que traducen los complejos lazos sociales y políticos entre la puna y los valles (Lazzari 1998, 1999).

En el caso del Período de Desarrollos Regionales o Tardío se plantea para el sur del Valle Yocavil el surgimiento de la diferenciación social y la jerarquización en el seno de la cultura santamariana, que se traduce especialmente en la producción especializada de bienes de prestigio, como los metales, y la exquisitez de la manufactura de sus piezas cerámicas (González y Tarragó 2004). Una observación que refuerza la complejidad de la sociedad santamariana es el grado de formalización de las prácticas ceremoniales que se visualiza mediante un patrón de asentamiento jerarquizado (Tarragó 1987, 1995) con sectores funcionalmente distintos y separados.

En sus investigaciones en los Valles Calchaquíes, a los cuales pertenece el Valle de Yocavil, Chaparro (2009) sostiene que, en líneas generales, los sitios de la región presentan una producción generalizada, donde no se observan divisiones estrictas en las etapas de reducción, siendo las rocas foráneas aquéllas que exhiben una mayor inversión en la manufactura y una estandarización en sus tamaños. El consumo y la producción lítica se hallan asociados a contextos domésticos y residenciales, donde, por consiguiente, se destaca la presencia de herramientas relacionadas con la práctica agrícola y de molienda (Chaparro 2009). 


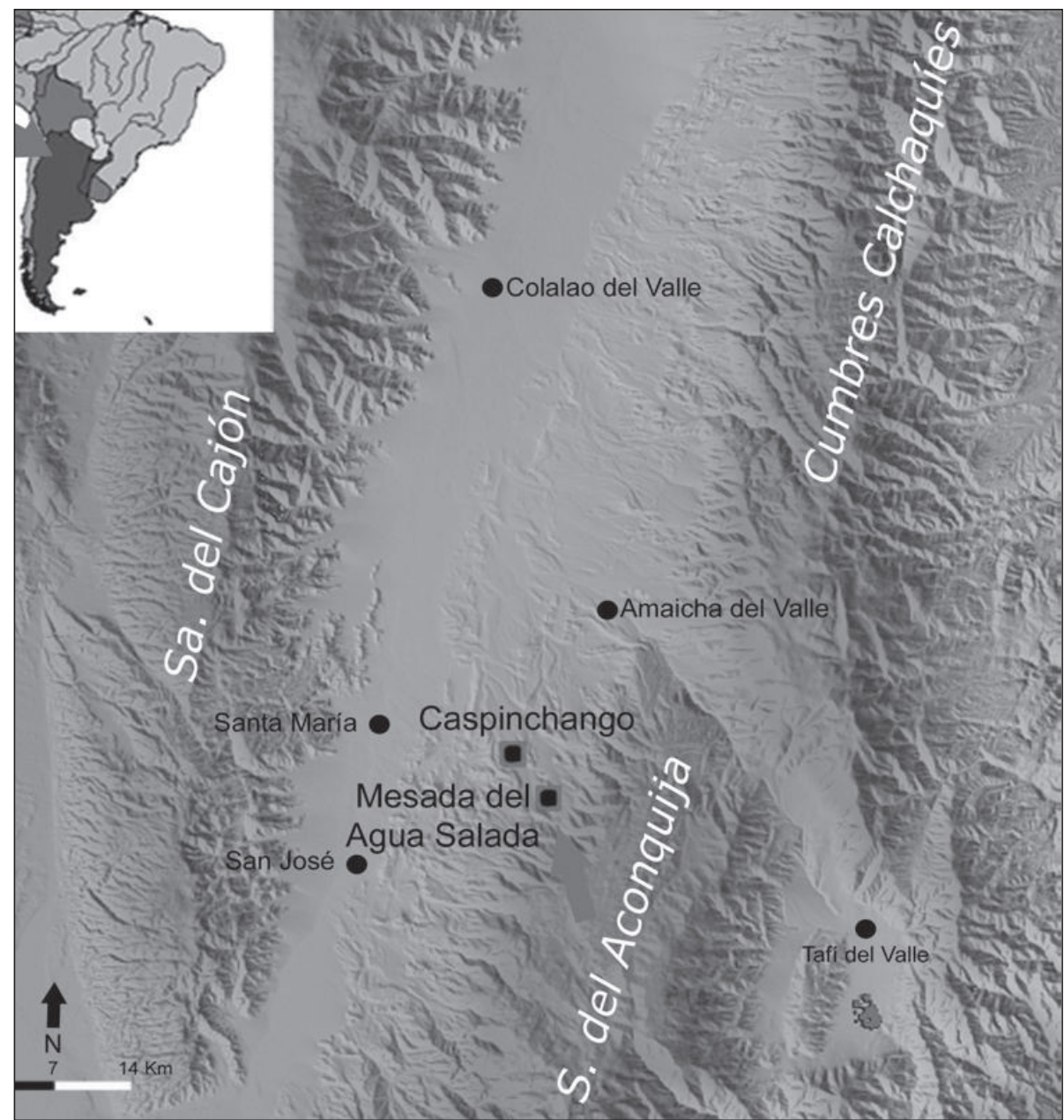

Figura 1: Localización de la localidad de Caspinchango en el Valle de Yocavil.

Los análisis sobre la organización tecnológica en todo el Noroeste Argentino durante el Período de Desarrollos Regionales, establecen que particularmente la obsidiana, por su valor excepcional para la talla, fue explotada intensivamente (Avalos 2003; Flores y Wynveldt 2003; Sprovieri 2007; Sprovieri y Baldini 2007), siendo las puntas de proyectil (dentro del enfoque teórico que considera el riesgo como una variable fundamental) producto de un planificación cuidadosa, que requeriría instrumentos confiables y transportables (Elías 2007).

El objetivo general de este trabajo se orienta a la primera de las características enunciadas anteriormente, es decir: indagar sobre la utilización de cada materia prima y obtener la descripción de las actividades realizadas en los recintos. Dicha meta se encuentra enmarcada en un proyecto general que abarca la sistematización de la base de recursos líticos y la definición de los sistemas de producción lítica de los sitios for- 
mativos del Valle de Yocavil (Carbonelli 2009b; sobre los sistemas de producción lítica, véase Ericson 1984). Para llevar a cabo el objetivo propuesto, utilizamos como herramienta el concepto de secuencia de producción. Entendemos por el mismo una forma de comprender la producción de instrumentos de piedra (Andrefsky 1998). En dicho sentido, nos adherimos a la definición de Aschero et al. (1995: 215): «la secuencia de producción comprende las distintas etapas de manufactura que fueron seguidas para producir artefactos de un determinado tipo de diseño y en una determinada clase de roca». Dicho proceso abarca la obtención de las materias primas, la extracción de formas base, su talla, el mantenimiento y, si existiere, el reciclaje.

\section{Procedencia y contexto de la muestra}

La localidad de Caspinchango se encuentra localizada en el Valle de Yocavil (Catamarca) (Figura 1). Ha sido objeto de investigaciones desde comienzos del siglo XX, a partir de los trabajos de Debenedetti y Weiser (Debenedetti 1921). Posteriormente, el equipo dirigido por Cigliano (1960) profundizó las indagaciones llevadas a cabo por sus predecesores y amplió la región de estudio. Los estudios de Tarragó y su equipo (1987) establecieron que las ocupaciones tardías de Caspinchango habrían funcionado como productoras de alimentos para los centros de mayor jerarquía de la región. Eran grandes áreas ocupadas por aldeas pequeñas compuestas por núcleos de viviendas circulares rodeadas de sus parcelas de cultivo (Tarragó 1999: 308).

La muestra de material lítico que aquí analizamos fue recuperada en dos recintos, con planta subcircular/cuadrangular, que forman parte de sendos conjuntos arquitectónicos de tipo habitacional detectados en la Mesada del Agua Salada (Lanzelotti et al. 2010). Se trata de una meseta de 101 ha ubicada entre los 2.600 y los $3.000 \mathrm{msnm}$ unos $4 \mathrm{~km}$ río arriba del poblado actual de Caspinchango (Lanzelotti et al. 2010).

El primer recinto, C48 R3, forma parte del conjunto denominado Unidad C48, la cual está compuesta por un patio central y dos recintos adosados, ajustándose al patrón arquitectónico conocido como Tafí típico. Dentro de los materiales recuperados en él podemos mencionar el conjunto cerámico, asignable al Período Formativo de la Argentina. En el conjunto, son abundantes los fragmentos de Alfarería Plomiza Pulida (Lanzelotti 2012). El recinto posee un fechado por AMS, sobre un fragmento de vértebra de un artiodáctilo recuperado en el piso de ocupación, de $1394 \pm 39$ AP, calibrado a dos sigmas.

El segundo, C88 R2, forma parte de la Unidad C88, que corresponde a un conjunto compuesto por una represa - una construcción con la función de almacenar agua para su posterior uso- y estructuras adosadas. A diferencia de la unidad anterior, en el recinto 2 de la unidad C88 se registró una mayor diversidad de fragmentos cerámicos: en la unidad estratigráfica superior (el relleno del recinto) se recuperaron materiales de cronología tardía (Santamariano, Negro sobre Rojo, y Ordinario peinado) y de momentos incaicos (Inca mixto), pero también materiales asignables al Temprano o al Temprano/Medio - primer mileno d.C.- (Ante pulido y alisado, Marrón y Naranja pulidos, Alfarerías Plomizas pulidas, Rojo pulido). En el nivel de ocupación se observa una situación similar: los estilos tempranos (Marrón pulido, 
Alfarerías Plomizas pulidas) coexisten con estilos tardíos (Santamariano y Negro sobre Rojo) (Lanzelotti 2012). Este nivel de ocupación se fechó por AMS, sobre una falange de un artiodáctilo que arrojó una edad de $642 \pm 42 \mathrm{AP}$, calibrado mediante dos sigmas. Hasta el momento, se considera que la Unidad C88 se construyó sobre la base de una unidad temprana del tipo Tafí, que fue remodelada en el Tardío, a partir de la construcción de recintos de planta cuadrangular (Lanzelotti 2012).

\section{Material y metodología}

Las muestras de material lítico recuperadas en los dos recintos $-\mathrm{C} 48 \mathrm{R} 3(\mathrm{n}=327)$ y C88 R2 ( $\mathrm{n}=119$ ) - fueron analizadas de forma separada, ya que provienen de dos excavaciones distintas. Una vez analizados, los datos en conjunto permitirán hablar de tendencias sobre la tecnología lítica del sitio Mesada del Agua Salada.

En primer lugar, antes de efectuarse el análisis tipológico de los materiales líticos, procedimos a la identificación petrográfica de los distintos tipos de materias primas, utilizando como muestra de referencia los cortes delgados realizados con anterioridad en las localidades de Andalhuala, Entre Ríos y Ampajango (Carbonelli 2009).

En segundo lugar, llevamos a cabo el análisis de los artefactos líticos, utilizando los criterios técnico-morfológicos de Aschero $(1975,1983)$ y Aschero y Hocsman (2004). En lo relativo a la etapa de obtención de materias primas para la talla, se tuvieron en cuenta las características de las formas base, los tamaños y la morfología de los núcleos recuperados. Por otra parte, en relación con la etapa de extracción de formas base, se analizaron las dimensiones relativas, los módulos de largo-ancho y el espesor de las mismas, comparándolos con las de los desechos de talla.

Para el proceso de talla observamos la categoría de clase técnica (Aschero y Hocsman 2004). Dicho concepto permite distinguir la inversión de trabajo puesta en juego a la hora de manufacturar un artefacto. Esto se logra mediante la medición de «la superposición de lascados que cubren total o parcialmente la superficie de una cara o las dos caras de un artefacto» (Hocsman y Escola 2006-07: 3). De esta manera se considera, por ejemplo, que un artefacto que presenta reducción bifacial, presenta mayor inversión de trabajo que otro artefacto que sólo vio afectado por conformación de su borde en una cara ${ }^{1}$.

Por otro lado, observamos la serie técnica (profundidad y extensión de los lascados), la situación de los lascados, la forma de los lascados sobre la cara y los rastros complementarios (Aschero 1975, 1983) de los instrumentos. En el caso de los desechos de talla, observamos las dimensiones absolutas y relativas, el origen de las extracciones, la presencia de talones y sus atributos.

En referencia, por último, a la etapa de mantenimiento se observaron los ángulos del bisel (real, estimado y de desgaste) y la presencia de lascas de reactivación.

\footnotetext{
1 Hocsman y Escola (2006-07) establecen el siguiente gradiente de esfuerzo tecnológico: artefactos con adelgazamiento bifacial, artefactos con reducción bifacial, artefactos con adelgazamiento unifacial, artefactos con reducción unifacial, artefactos con trabajo no invasivo bifacial, artefactos con trabajo no invasivo unifacial, artefactos con trabajo no invasivo alternante, artefactos con trabajo bipolar.
} 


\section{Resultados obtenidos}

Del total de los artefactos recuperados, en el caso del recinto C48 R3 el 38\% son andesitas, mientras que en C88 R2 la materia prima más representada es el cuarzo, con un $73 \%$ (Cuadro 1). El porcentaje de cuarzo en el primer recinto también es importante, alcanzando el 35\%, mientras que el porcentaje de andesitas en el recinto C88 R2 apenas alcanza el 9\%. En estos valores, resulta llamativo el elevado porcentaje alcanzado por el cuarzo, una materia prima considerada de mala calidad por su alta tenacidad.

Cuadro 1: Distribución de las materias primas en los recintos C48 R3 (N=327) y C88 R2 (N=119)

\begin{tabular}{lrrrr}
\hline \multicolumn{1}{c}{ Materias primas } & \multicolumn{2}{c}{$\mathbf{C 4 8} \mathbf{R 3}$} & \multicolumn{2}{c}{$\mathbf{C 8 8 ~ R 2 ~}$} \\
& \multicolumn{1}{c}{$\mathbf{c}$} & $\mathbf{1}$ & \multicolumn{1}{c}{ n } & \% \\
\hline Andesita & 125 & 38 & 11 & 9 \\
Cuarcita & 9 & 3 & 6 & 5 \\
Cuarzo & 115 & 35 & 87 & 73 \\
Granito & 1 & 1 & 1 & 1 \\
Metamórfica & 69 & 21 & 11 & 9 \\
Obsidiana & 4 & 1 & 2 & 2 \\
Otras & 4 & 1 & 1 & 1 \\
Totales & 327 & 100 & 119 & 100 \\
\hline
\end{tabular}

Otro punto a destacar es la presencia en ambos recintos de rocas metamórficas, las cuales también son consideradas como no óptimas para la talla, siguiendo los criterios sugeridos por Aragón y Franco (1997), debido a que no presentan en general fractura concoide y al elevado porcentaje de fenocristales en su matriz. No obstante, al observar la distribución de materias primas por clases tipológicas en ambos recintos (Cuadros 2 y 3), podemos destacar, dada la elevada frecuencia de desechos de rocas metamórficas, que sobre dichas materias primas se efectuaron varias etapas de la secuencia de producción.

Cuadro 2: Distribución de materias primas por clases tipológicas en el recinto C48 R3 ( N=326)

\begin{tabular}{lcccrr}
\hline Materia prima & $\begin{array}{c}\text { Desechos de } \\
\text { talla }\end{array}$ & Núcleos & $\begin{array}{c}\text { Artefactos } \\
\text { tallados }\end{array}$ & $\begin{array}{c}\text { Artefactos } \\
\text { no tallados }\end{array}$ & Total \\
\hline Andesita & 117 & 0 & 7 & 0 & 125 \\
Cuarcita & 9 & 0 & 0 & 0 & 9 \\
Cuarzo & 111 & 1 & 3 & 0 & 114 \\
Granito & 1 & 0 & 0 & 0 & 1 \\
Metamórfica & 64 & 0 & 2 & 3 & 69 \\
Obsidiana & 3 & 0 & 1 & 0 & 4 \\
Otras & 4 & 0 & 0 & 0 & 4 \\
Total & 309 & 1 & 13 & 3 & 326 \\
\hline
\end{tabular}


Cuadro 3: Distribución de materias primas por clases tipológicas en el recinto C88 R2 ( $N=119)$

\begin{tabular}{lrccrr}
\hline Materia prima & $\begin{array}{c}\text { Desechos de } \\
\text { talla }\end{array}$ & Núcleos & $\begin{array}{c}\text { Artefactos } \\
\text { tallados }\end{array}$ & $\begin{array}{c}\text { Artefactos } \\
\text { no tallados }\end{array}$ & Total \\
\hline Andesita & 10 & 0 & 1 & 0 & 11 \\
Cuarcita & 6 & 0 & 0 & 0 & 6 \\
Cuarzo & 86 & 0 & 1 & 0 & 87 \\
Granito & 1 & 0 & 0 & 0 & 1 \\
Metamórfica & 11 & 0 & 0 & 0 & 11 \\
Obsidiana & 1 & 0 & 1 & 0 & 2 \\
Otras & 1 & 0 & 0 & 0 & 1 \\
Total & 116 & 0 & 3 & 0 & 119 \\
\hline
\end{tabular}

Otro de los elementos que se desprende del análisis es el hecho de que las materias primas utilizadas preferentemente para los artefactos tallados son las andesitas y las rocas metamórficas en el recinto C48 R3, mientras que en el recinto C88 R2, son las andesitas y el cuarzo. En ambas muestras, los artefactos que fueron producto de la talla y que utilizan como soporte obsidianas son puntas de proyectil.

Resulta llamativo el desequilibrio existente en ambos recintos entre la cantidad de desechos y la de artefactos tallados (Cuadro 4). Esta situación podemos atribuirla a diferentes causas, que no son mutuamente excluyentes. En primer lugar, es factible pensar que no se excavó en los recintos (dentro de la localidad de Caspinchango) en que se encuentra el mayor número de instrumentos. En segundo término, resulta posible también que las formas base de los instrumentos manufacturados fueran transportadas hacia otros recintos o sitios. Y en tercer lugar, existe la posibilidad de que, al igual que en otros sitios del Noroeste Argentino como Soria 2 (Carbonelli 2009a) y Tebenquiche 1 (Moreno 2005), las lascas con filo natural hayan sido utilizadas como instrumentos.

Cuadro 4: Grupos tipológicos en los recintos C48 R3 (N=17) y C88 R2 (N=3)

\begin{tabular}{lrrrr}
\hline \multicolumn{1}{c}{ Grupos tipológicos } & \multicolumn{2}{c}{ C48 R3 } & \multicolumn{2}{c}{ C88 R2 } \\
\hline & n & \multicolumn{1}{c}{ \% } & \multicolumn{1}{c}{ n } & \multicolumn{1}{c}{$\%$} \\
\hline Raspadores & 3 & 17,64 & 0 & 0 \\
Artefactos de talla sumaria & 6 & 35,29 & 1 & 33 \\
Raedera & 0 & 0 & 1 & 33 \\
Punta entre muescas & 1 & 5,88 & 0 & 0 \\
Filo natural con rastros complementarios & 1 & 5,88 & 0 & 0 \\
Punta de proyectil & 2 & 11,76 & 1 & 33 \\
Núcleos & 1 & 5,88 & 0 & 0 \\
Percutores & 2 & 11,76 & 0 & 0 \\
Cuchillo & 1 & 5,88 & 0 & 0 \\
Totales & 17 & 100 & 3 & 100 \\
\hline
\end{tabular}




\subsection{Obtención de las materias primas}

En lo que respecta a la determinación de las fuentes de aprovisionamiento, la misma se realizó con base en la información geológica disponible para el área (Ruiz Huidobro 1972), con el fin de localizar las fuentes más próximas al sitio factibles. Posteriormente, dicha información fue comprobada mediante prospecciones en el terreno y la comparación de las muestras de mano obtenidas con los cortes petrográficos efectuados en las zonas de Andalhuala, Ampajango y Entre Ríos (Carbonellli 2009a).

Con respecto a la distinción entre materias primas locales, seguimos la propuesta de Civalero y Franco (2003) y Cardillo y Nuviala (2004). Dichos autores han considerado materias primas locales aquéllas ubicadas dentro de un rango de $40 \mathrm{~km}$. Dentro de estas, son inmediatamente locales aquéllas con fuentes en un rango de hasta 5 $\mathrm{km}$, locales cercanas aquellas entre 5 y $20 \mathrm{~km}$, locales lejanas aquellas entre 20 y 40 $\mathrm{km}$. No locales son las materias primas con fuentes a más de $40 \mathrm{~km}$.

Al comparar la oferta local de materias primas líticas con las rocas efectivamente seleccionadas para la manufactura de artefactos en los dos recintos analizados del sitio Mesada del Agua Salada, podemos afirmar que estas últimas se encuentran en el espacio inmediatamente disponible al sitio. Esto último se desprende de la existencia de las siguientes fuentes próximas a él:

a) Afloramiento de la formación Yasyamayo: disponible a $5 \mathrm{~km}$ del sitio. Está conformado por rodados de andesitas, basandesitas y otras vulcanitas, en forma de conglomerados partidos. Es frecuente observar en él la presencia de bloques de cuarzo, de entre 10 y $50 \mathrm{~cm}$.

b) Formación La Mesada: situada entre los ríos Entre Ríos y Andalhuala, a una altura de 3.500 metros. Está compuesta por un fanglomerado de rodados de rocas del basamento precámbrico, en su mayoría rocas metamórficas.

c) Formación Las Salinas: tiene una litología similar a la Formación La Mesada y su superficie está conformada por las formaciones terciarias plegadas y fracturadas. Parte de su superficie se cuenta en la zona de Caspinchango, La Maravilla más precisamente, que se encuentra a una distancia de entre 5 y $10 \mathrm{~km}$ del sitio.

d) Formación Caspinchango: ubicada en el tercer nivel pedemontano, se conserva en una amplia superficie que va desde los bloques montañosos del Aconquija y de la Sierra del Cajón. Sobre ella se ubica el sitio Mesada del Agua Salada. Este extenso depósito cuartario se halla constituido en gran parte por rodados de rocas volcánicas, del basamento precámbrico, mayoritariamente andesitas-basaltos, provenientes de los afloramientos de las Formaciones Las Arcas y Los Corrales. Su calidad para la talla varía de regular a buena, observando el espectro de pátinas, cortezas e inclusiones.

Consideramos que las cuarcitas, de buena calidad para la talla por su textura y fractura, pertenecen al grupo de las rocas locales cercanas (entre 5 y $20 \mathrm{~km}$ del sitio). Se distribuyen en el paisaje en forma de concreciones, en las Sierras del Aconquija.

Por último, hemos efectuado análisis de procedencia para el caso de las obsidianas, con la finalidad de detectar cuáles han sido las fuentes de obsidiana de donde procede el material. Las muestras fueron analizadas por el Dr. Glascock en el Missouri 
University Research Reactor (MURR) por medio de Fluorescencia de Rayos X de Energía Dispersiva (EDXRF) y se detectó la representación de dos fuentes: Ona-Las Cuevas, para el recinto C48, y Laguna Cavi, para el recinto C88. Ambas fuentes se encuentran en la Puna Argentina y ya han sido investigadas (Escola y Hocsman 2007; Yacobaccio et al. 2004). Por lo tanto, la obsidiana se revela como el único recurso alóctono (con fuentes situadas a más de $40 \mathrm{~km}$ del sitio) utilizado por estos grupos agro-pastoriles. A la par, esta materia prima es la de mejor calidad para la talla, designándosela como excelente por estructura vítrea y filo.

Como primera conclusión, al considerar la distancia de las materias primas al sitio, podemos decir que dentro del grupo de las rocas inmediatamente locales encontramos el cuarzo, el granito, las andesitas y las rocas metamórficas. En el grupo de las locales cercanas a la cuarcita y como alóctonas o no locales se encuentra la obsidiana. Si retomamos lo observado en el Cuadro 1, podemos establecer que en el sitio Mesada del Agua Salada se aprovecharon casi exclusivamente rocas inmediatamente locales, en menor medida rocas locales cercanas, y en un ínfimo porcentaje, las alóctonas.

En referencia a la variable accesibilidad de las materias primas, nos inclinamos a pensar (luego de haber efectuado prospecciones) que los nódulos, núcleos de la rocas inmediatamente locales, pudieron haber sido obtenidos de las fuentes primarias, pero principalmente de las fuentes secundarias, como el río Caspinchango o los cauces secos de sus afluentes y arroyos. Allí se encuentran disponibles rodados de considerable tamaño (50 $\mathrm{cm}$ aproximadamente) de cuarzos, metamórficas y andesitas.

Pasando ahora al análisis de la muestra de artefactos líticos recuperados en los recintos C48 R3 y C88 R2, la etapa de obtención de materias primas líticas se halla únicamente representada por un nódulo de granito en el primero de los recintos. Sugerimos que, dada la cercanía a las fuentes secundarias de materias primas, es factible pensar que las primeras etapas de reducción se realizarían allí.

\subsection{Extracción de formas base}

La comparación de las cantidades de desechos de talla y de núcleos evidencia una nítida desproporción en favor de los primeros. El único núcleo encontrado en el recinto C48 R3 se corresponde con un núcleo pequeño de lascados aislados de cuarzo. Esta baja frecuencia puede ser atribuida a la escasa superficie excavada, pero también cabe pensar que, como las fuentes de materias primas se hallaban cercanas, la mayor parte de las actividades de reducción primaria no se habrían efectuado en el sitio. Dentro de la muestra del recinto C48 R3 se incluyen, además, dos percutores de roca metamórfica. Siguiendo a Hocsman et al. (2003), creemos que una posible explicación de la escasez de percutores es que éstos hayan sido utilizados indistintamente también como núcleos y que hayan sido descartados en otros recintos.

Para completar esta sección, analizaremos las formas bases de los artefactos tallados. Es en el recinto C48 R3 (ver Cuadro 2) donde encontramos el mayor número de estos artefactos. En todos los casos, la forma base utilizada son las lascas angulares. Dicho patrón se repite de la misma forma en el recinto C88 R2, donde el número de artefactos producto de la talla es de 3 (ver Cuadro 3). Como ya se señaló más arriba, 
las materias primas utilizadas preferentemente para los artefactos son las andesitas y las rocas metamórficas en el recinto C48 R3, y las andesitas y el cuarzo en el recinto C88 R2.

$\mathrm{Al}$ analizar las propiedades de las formas base de los instrumentos (Cuadro 5), encontramos diversidad en los módulos de tamaño y espesores relativos empleados en el recinto C48 R3, desde las lascas muy pequeñas hasta las medianas grandes y desde las lascas muy delgadas hasta las gruesas. Al observar el módulo de largo-ancho encontramos que predominan las formas base alargada y mediano-normal. En el recinto C88 R2, donde la muestra es mucho menor $(n=3)$, encontramos un comportamiento similar en la elección de los tamaños, módulos de largo-ancho y espesores de las formas bases. Esto nos indicaría la ausencia de estandarización de las formas base en la tecnología lítica de Mesada del Agua Salada.

Cuadro 5: Propiedades de las formas base de los instrumentos enteros en los recintos C48 R3 $(\mathrm{N}=9)$ y C88 R2 $(\mathrm{N}=3)$

\begin{tabular}{llrrrr}
\hline & \multicolumn{2}{c}{ C48 R3 } & \multicolumn{2}{c}{ C88 R2 } \\
\hline Tamaños relativos & n & \% & n & \multicolumn{1}{c}{$\%$} \\
& Muy pequeñas & 1 & 11,11 & 1 & 33,33 \\
& Pequeñas & 4 & 44,44 & 0 & 0 \\
& Medianas pequeñas & 3 & 33,34 & 2 & 66,67 \\
& Medianas grandes & 1 & 11,11 & 0 & 0 \\
& Grandes & 0 & 0 & 0 & 0 \\
& Muy grandes & 0 & 0 & 0 & 0 \\
& Totales & 9 & 100 & 3 & 100 \\
\hline Módulo largo-ancho & Laminares normales & 0 & 0 & 0 & 0 \\
& Medianas alargadas & 4 & 44,44 & 0 & 0 \\
& Medianas normales & 5 & 55,56 & 3 & 100 \\
& Cortas anchas & 0 & 0 & 0 & 0 \\
& Cortas muy anchas & 0 & 0 & 0 & 0 \\
& Cortas anchísimas & 0 & 0 & 0 & 0 \\
& Totales & 9 & 100 & 3 & 100 \\
\hline Espesores relativos & Muy delgadas & 3 & 33,34 & 1 & 33,33 \\
& Delgadas & 2 & 22,22 & 1 & 33,33 \\
& Gruesas & 4 & 44,44 & 1 & 33,33 \\
& Muy gruesas & 0 & 0 & 0 & 0 \\
& Totales & 9 & 100 & 3 & 100 \\
\hline
\end{tabular}

Al comparar los datos de las formas base con los atributos de los desechos de talla (Cuadro 6), los tamaños que tienen los desechos factiblemente podrían haber sido utilizados como formas base de instrumentos, ya que coinciden con el módulo de tamaño de éstos. Al observar la categoría de módulo de largo-ancho, encontramos que se repite la coincidencia entre desechos e instrumentos. Es dable pensar que en ambos recintos, las lascas mediano-normales y de espesores muy delgados y delgados hayan sido formas base que fueron descartadas. No obstante, al observar los altos porcentajes de lascas pequeñas $(49,25 \%)$ y muy pequeñas $(37,31 \%)$ en el recinto C48 R3 y muy pequeñas $(74,01 \%)$ y pequeñas $(22,23 \%)$ en el recinto C 88 R2, sugerimos 
que podría haber existido una tendencia a la búsqueda de lascas grandes como formas base, y que los desechos de talla restantes serían el producto de tareas de talla o el remanente de las tareas de extracción.

En síntesis, podemos pensar que a pesar del bajo número de núcleos y percutores en ambos recintos, la existencia de potenciales formas base entre los desechos de talla nos indican la presencia de las primeras etapas de la secuencia de producción.

Cuadro 6: Atributos de los desechos de talla en los recintos C48 R3 (N=309) y C88 R2 (N=116)

\begin{tabular}{|c|c|c|c|c|c|}
\hline & & \multicolumn{2}{|c|}{ C48 R3 } & \multicolumn{2}{|c|}{ C88 R2 } \\
\hline & & $\mathbf{n}$ & $\%$ & $\mathbf{n}$ & $\%$ \\
\hline \multirow[t]{7}{*}{ Tamaños relativos } & Muy pequeñas & 25 & 37,31 & 20 & 74,07 \\
\hline & Pequeñas & 33 & 49,25 & 6 & 22,23 \\
\hline & Medianas pequeñas & 7 & 10,46 & 1 & 3,7 \\
\hline & Medianas grandes & 2 & 2,98 & 0 & 0 \\
\hline & Grandes & 0 & 0 & 0 & 0 \\
\hline & Muy grandes & 0 & 0 & 0 & 0 \\
\hline & Totales & 67 & 100 & 27 & 100 \\
\hline \multirow[t]{8}{*}{ Módulo largo-ancho } & Laminar-angosta & 1 & 1,49 & 2 & 7,4 \\
\hline & Laminares normales & 6 & 8,95 & 3 & 11,11 \\
\hline & Medianas alargadas & 6 & 8,95 & 2 & 7,4 \\
\hline & Medianas normales & 26 & 38,8 & 8 & 29,62 \\
\hline & Cortas anchas & 12 & 17,91 & 5 & 18,51 \\
\hline & Cortas muy anchas & 14 & 20,89 & 4 & 14,81 \\
\hline & Cortas anchísimas & 2 & 2,98 & 3 & 11,11 \\
\hline & Totales & 67 & 100 & 27 & 100 \\
\hline \multirow[t]{5}{*}{ Espesores relativos } & Muy delgadas & 96 & 31,06 & 57 & 49,13 \\
\hline & Delgadas & 152 & 49,19 & 40 & 34,48 \\
\hline & Gruesas & 55 & 17,79 & 18 & 15,51 \\
\hline & Muy gruesas & 6 & 1,94 & 1 & 0,86 \\
\hline & Totales & 309 & 100 & 116 & 100 \\
\hline \multirow{9}{*}{$\begin{array}{l}\text { Tipos de talones } \\
\text { (enteros o parcial- } \\
\text { mente fracturados) }\end{array}$} & Corticales & 8 & 4,41 & 3 & 4,28 \\
\hline & Lisos-naturales & 0 & 0 & 0 & 0 \\
\hline & Lisos & 88 & 45,59 & 42 & 60 \\
\hline & Diedros & 7 & 3,62 & 2 & 2,88 \\
\hline & Facetados & 4 & 2,07 & 8 & 11,42 \\
\hline & Filiformes & 4 & 2,07 & 0 & 0 \\
\hline & puntiformes & 2 & 1,03 & 1 & 1,42 \\
\hline & No diferenciados & 80 & 41,45 & 14 & 20 \\
\hline & Totales & 193 & 100 & 70 & 100 \\
\hline
\end{tabular}

\subsection{Proceso de talla}

Tomando en consideración el total de las muestras analizadas, podemos encontrar diferencias en el estado de fragmentación de los desechos de talla de los recintos. A través del gráfico de la Figura 2 apreciamos que, en el recinto C48 R3, el 5\% de las lascas son indiferenciadas, el $25,59 \%$ corresponden a lascas fracturadas con talón y 


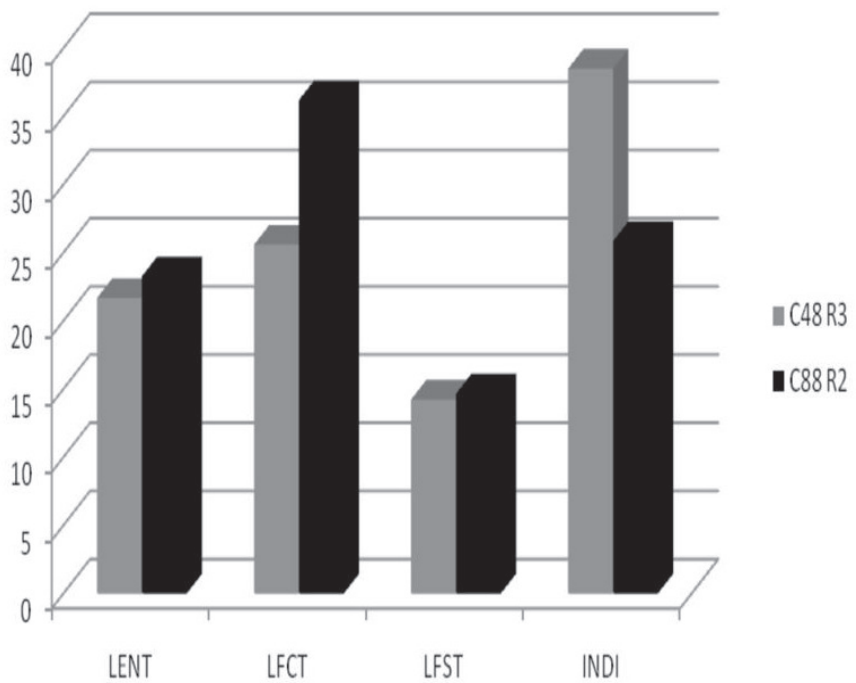

Figura 2: Estado de fragmentación de los desechos de talla en C48 R3 (N=309) y C88 R2 (N=116).

(LENT: Lascas enteras; LFCT: lascas fracturadas con talón; LFST: lascas fracturadas sin talón; INDI: indiferenciados).

el $21,68 \%$ a las lascas enteras. El panorama se completa con un 14, $23 \%$ de lascas fracturadas sin talón. En el recinto C88 R2, la situación es algo diferente: el mayor porcentaje corresponde a las lascas fracturadas con talón (36,2\%), seguidas por las lascas indiferenciadas $(25,86 \%)$.

Dado el escaso número de núcleos encontrados en el recinto C48 R3 (n=1), y el nulo registro de núcleos en el recinto C88 R2, ambas muestras pueden ser explicadas, siguiendo a Sullivan y Rozen (1985), como el resultado de eventos donde predomina la manufactura de herramientas. Esta afirmación se corrobora al analizar una vez más el módulo de tamaño en los desechos de talla (Cuadro 6). El predominio en el recinto C48 R3 de las lascas pequeñas (49,25\%), y en el recinto C88 R2 de las lascas muy pequeñas $(74,07 \%)$, indica, en los dos casos, que la mayor parte de las actividades vinculadas a la producción lítica se focalizarían en dar forma y regularizar los filos de los instrumentos (Bellelli 1991; Espinosa 1995, 1996; Hocsman et.al 2003).

En relación con el bajo número de artefactos tallados, el Cuadro 4 muestra que la diversidad de grupos tipológicos en ambos recintos es restringida, particularmente en el recinto C88 R2. En dicho sentido, destacamos la presencia, en el recinto C48 R3, de un 35,29\% de artefactos de talla sumaria ${ }^{2}$ y un 5,29 \% de filos naturales con rastros complementarios, lo cual indica que, al igual que en el caso de Soria 2 (Carbonelli 2009a, 2011), las poblaciones formativas privilegiarían el trabajo sobre los filos y no sobre las caras de los artefactos. Llevando más lejos el análisis y teniendo en consideración la elevada frecuencia de lascas, posibles soportes, desechadas, podemos retomar aquí la hipótesis del uso directo de los filos naturales.

2 Por talla sumaria entendemos aquellos artefactos cuyo filo es producto de escasos lascados, que afectan sólo al borde y no al cuerpo de la pieza. 


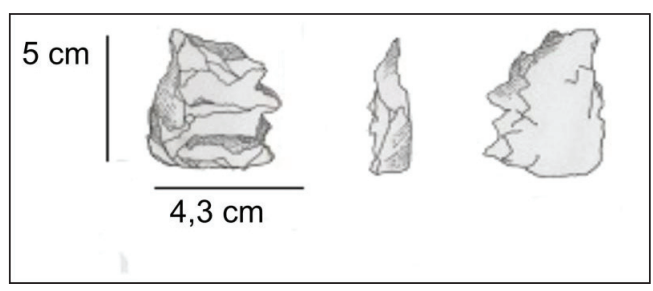

Figura 3: Raedera de cuarzo.

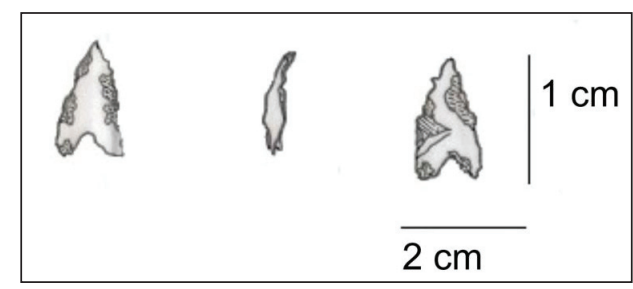

Figura 4: Punta de proyectil de obsidiana, apenduculada de base escotada (B1).

En el recinto C48 R3 (ver Cuadro 2), podemos apreciar que la andesita es la materia prima de mayor representación en la clase tipológica de artefactos tallados. Dicha proporción se mantiene en el resto de las clases tipológicas. Los únicos artefactos que conforman un producto de la talla son los filos naturales con rastros complementarios y dos percutores, cuyo soporte son rocas metamórficas (ver Cuadro 7). En el recinto C88 R2 encontramos 3 artefactos tallados: una raedera de cuarzo (Figura 3), un artefacto de talla sumaria de andesita y una punta de proyectil de obsidiana (Figura 4). No se registran en este caso artefactos no tallados.

Cuadro 7: Distribución de materias primas por grupo tipológicos en el recinto C48 R3 ( $N=16)$

\begin{tabular}{lcccccccc}
\hline \multicolumn{1}{c}{$\begin{array}{c}\text { Materia } \\
\text { prima }\end{array}$} & $\begin{array}{c}\text { Artefactos } \\
\text { de talla } \\
\text { sumaria }\end{array}$ & $\begin{array}{c}\text { Puntas de } \\
\text { proyectil }\end{array}$ & FNRC & Raspador & Percutor Cuchillo & $\begin{array}{c}\text { Puntas } \\
\text { entre } \\
\text { muescas }\end{array}$ & $\begin{array}{c}\text { Tota- } \\
\text { les }\end{array}$ \\
\hline Andesita & 3 & 1 & 0 & 1 & 0 & 1 & 1 & 7 \\
Cuarzo & 2 & 0 & 0 & 1 & 0 & 0 & 0 & 3 \\
Metamórfica & 1 & 0 & 1 & 1 & 2 & 0 & 0 & 5 \\
Obsidiana & 0 & 1 & 0 & 0 & 0 & 0 & 0 & 1 \\
Total & 6 & 2 & 1 & 3 & 2 & 1 & 1 & 16 \\
\hline
\end{tabular}

FNRC: Filo natural con rastros complementarios

Como ya se indicó más arriba, para evaluar el grado de inversión de energía en el proceso de talla de los instrumentos correspondientes recurrimos a la categoría de Clase Técnica (Aschero y Hocsman 2004), implicando los distintos costos de producción diferentes grados de conocimientos prácticos, experiencia y destreza manual por parte de los talladores (Hocsman y Escola 2006-07).

Todos los artefactos tallados de ambos sitios, a excepción de las puntas de proyectil de obsidiana, presentan un trabajo no invasivo o marginal sobre una de las caras. A través de estas características es dable pensar que el trabajo sobre los filos y las superficies de los instrumentos no fue intenso.

Las puntas de proyectil de obsidiana son los únicos artefactos que revisten un mayor esfuerzo tecnológico. Esto se demuestra en la búsqueda de una morfología de filo particular (Hocsman y Escola 2006-07), con un determinado ángulo y forma de bisel, delineación y forma geométrica. En este caso, los lascados afectan sólo a los bordes de la pieza, sin alcanzar las zonas centrales, siendo marginales al cubrir menos del 50\% de la cara (Aschero 1975). Sobre la forma base (lascas no diferenciadas) se aplicó la técnica de reducción bifacial. En otras palabras, las piezas se manufactura- 
ron mediante lascados bifaciales, que afectaron proporcionalmente más a las caras que a los bordes, sin llegar a superponerse con los lascados que provienen del otro borde (Hocsman y Escola 2006-07). A través de esta actividad de talla, se redujo el espesor de las mismas y se logró el objetivo de los talladores de obtener un contorno geométrico específico en los artefactos (Hocsman y Escola 2006-07).

Morfológicamente, las puntas de proyectil de obsidiana se corresponden con dos de los subgrupos definidos por Escola (1987): una de ellas es una punta de proyectil apedunculada de base escotada (B1) y otra es una punta de proyectil con pedúnculo diferenciado y aletas entrantes (A.1.I). Fueron los mismos subgrupos tipológicos encontrados en Soria 2 (Carbonelli 2009a, 2010), lo que evidenciaría la distribución de dichas morfologías en todo el Valle de Yocavil.

La información obtenida sobre la talla de los instrumentos se ve respaldada al analizar los talones de los desechos de talla (Cuadro 6). En ambos recintos predominan los talones lisos, alcanzando un 45,59\% en el C48 R3 y un $60 \%$ en el recinto C88 R2. Esto nos permite subrayar dos puntos: en primer término demuestra que la percusión ha sido la técnica utilizada preferentemente en la secuencia de producción, y, en segundo lugar, que se optó por preparar las plataformas, despojándolas de corteza.

Por otro lado, el bajo porcentaje de talones facetados es coherente con la ausencia de reducción y adelgazamiento bifacial (Bellelli y Kligmann 1996; Nami y Bellelli 1994) y la escasa talla registrada en la mayoría de los instrumentos. El bajo porcentaje de talones filiformes, puntiformes y diedros nos habla del escaso uso de la técnica de presión en los primeros (Espinosa 1995; Valverde 2003) y de etapas avanzadas de reducción en los segundos (Elías 2007).

Los talones filiformes pueden haber sido generados por la manufactura de instrumentos mediante la técnica de reducción bifacial (Espinosa 1995). El bajo porcentaje de talones corticales, $8 \%$ en un recinto y $3 \%$ en otro, coincide con la escasez de núcleos. De esta forma, robustece el enunciado anterior sobre la falta de representación de las primeras etapas de la reducción lítica en las muestras que analizamos.

$\mathrm{Al}$ analizar las frecuencias del origen de las extracciones (Figura 5) observamos que en ambos recintos predominan las lascas internas (64,4\% en el recinto C48 R3 y 74,13\% en el recinto C88 R2), lo que refuerza nuestra aserción anterior sobre el esfuerzo invertido en las tareas de conformación y regularización de filos (Bellelli 1991; Escola 2000; Valverde 2003).

Al momento de analizar en forma divergente el origen de las extracciones en los recintos, en el C48 R3 (Cuadro 8) encontramos que en las materias primas de mayor representación (andesitas, cuarzos y metamórficas) la frecuencia de lascas no diferenciadas es considerable. Creemos que esto se debe a la calidad regular y mala de numerosos desechos de las materias primas locales. Sin embargo, esta no constituyó una variable importante a la hora de decidir sobre cuál de las materias primas locales se trabajaría. El bajo número de lascas de cuarcita, de mejor calidad que el cuarzo, las andesitas y las metamórficas apoya esta idea.

El cuarzo es la materia prima que aglutina el mayor número de lascas externas y de reactivación de núcleos, a partir de lo cual es dable pensar que los núcleos de cuarzo hayan ingresado en un número pequeño (acorde con la muestra de desechos), siendo reducidos en el recinto. Siguiendo el mismo razonamiento, el escaso porcentaje de 
lascas de obsidiana lleva a inferir que los instrumentos ingresaron enteros en el recinto.

En referencia al recinto C88 R2 (Cuadro 9), el cuarzo concentra la mayor diversidad de tipos de lascas, siendo las angulares las de mayor representación ( $\mathrm{n}=59)$, lo que muestra el marcado énfasis en la regularización de filos. Siguen en casi el mismo porcentaje dos materias primas locales: las andesitas y las metamórficas. Las lascas angulares y no diferenciadas encontradas en dichas materias primas reflejan la misma etapa de producción que el cuarzo.

Cuadro 8: Origen de las extracciones en el recinto C48 R3 ( $\mathrm{N}=309)$

\begin{tabular}{|c|c|c|c|c|c|c|c|}
\hline & Andesita & Cuarcita & Cuarzo & Metamórfica & Obsidiana & Otras & Total \\
\hline Lasca angular & 62 & 8 & 44 & 25 & 2 & 1 & 142 \\
\hline Lasca con dorso & 1 & 1 & 0 & 0 & 0 & 0 & 2 \\
\hline $\begin{array}{l}\text { Lasca de adelgazamiento } \\
\text { bifacial }\end{array}$ & 1 & 0 & 1 & 0 & 0 & 0 & 2 \\
\hline Lasca de arista & 18 & 0 & 2 & 6 & 1 & 0 & 27 \\
\hline Lasca de arista doble & 0 & 0 & 1 & 0 & 0 & 0 & 1 \\
\hline Lasca de flanco de núcleo & 0 & 0 & 2 & 0 & 0 & 0 & 2 \\
\hline Lasca de reducción bifacial & 0 & 0 & 1 & 0 & 0 & 0 & 1 \\
\hline Lasca en cresta & 1 & 0 & 0 & 0 & 0 & 0 & 1 \\
\hline Lasca no diferenciada & 18 & 0 & 40 & 25 & 0 & 2 & 85 \\
\hline Lasca nodular & 1 & 0 & 0 & 0 & 0 & 0 & 1 \\
\hline Lasca plana & 9 & 0 & 7 & 5 & 0 & 1 & 22 \\
\hline Lasca primaria & 5 & 0 & 6 & 2 & 0 & 0 & 13 \\
\hline Lasca reactivación directa & 0 & 0 & 5 & 0 & 0 & 0 & 5 \\
\hline Lasca secundaria & 1 & 0 & 1 & 1 & 0 & 0 & 3 \\
\hline Lasca tableta de núcleo & 1 & 0 & 1 & 0 & 0 & 0 & 2 \\
\hline Total & 118 & 9 & 111 & 64 & 3 & 4 & 309 \\
\hline
\end{tabular}

Cuadro 9: Origen de las extracciones en el recinto C88 R2 ( $\mathrm{N}=116)$

\begin{tabular}{lccccccr}
\hline & Andesita & Cuarcita & Cuarzo & Metamórfica & Obsidiana & Otras & Total \\
\hline Lasca angulares & 9 & 2 & 53 & 4 & 0 & 1 & 69 \\
Lasca con dorso & 0 & 0 & 2 & 0 & 0 & 0 & 2 \\
Lasca de adelgazamiento & 0 & 0 & 0 & 0 & 0 & 1 & 1 \\
bifacial & 1 & 1 & 5 & 0 & 0 & 0 & 7 \\
Lasca de arista & 0 & 0 & 0 & 0 & 0 & 0 & 0 \\
Lasca de arista doble & 0 & 0 & 2 & 0 & 0 & 0 & 2 \\
Lasca de flanco de núcleo & 0 & 0 & 0 & 0 & 0 & 0 & 0 \\
Lasca de reducción bifacial & 0 & 0 & 0 & 0 & 0 & 0 & 0 \\
Lasca en cresta & 0 & 0 & 8 & 6 & 1 & 0 & 15 \\
Lasca no diferenciada & 0 & 0 & 0 & 0 & & 0 & 0 \\
Lasca nodular & 0 & 1 & 5 & 1 & 0 & 0 & 7 \\
Lasca plana & 0 & 0 & 4 & 0 & 0 & 0 & 4 \\
Lasca primaria & 0 & 0 & 3 & 0 & 0 & 0 & 3 \\
Lasca reactivación directa & 0 & 1 & 1 & 0 & 0 & 0 & 2 \\
Lasca secundaria & 0 & 1 & 3 & 0 & 0 & 0 & 4 \\
Lasca tableta de nucleo & 10 & 6 & 86 & 11 & 1 & 2 & 116 \\
Total & & & & & & & \\
\hline
\end{tabular}




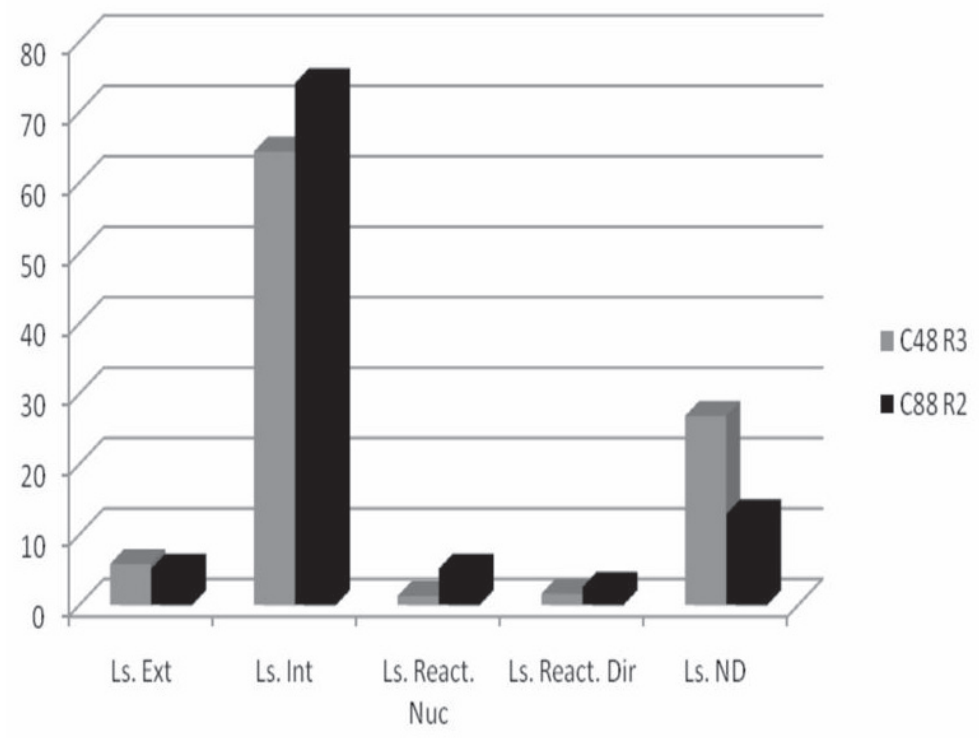

Figura 5: Origen de las extracciones en $\mathrm{C} 48 \mathrm{R} 3(\mathrm{~N}=309)$ y C88 R2 (N=116). (Ls. Ext: lascas externas; Ls.Int: lascas internas, Ls. React. Nuc.: lascas de reactivación de núcleo, Ls. React. Dir: lascas de reactivación directa; Ls. ND: lascas no diferenciadas).

\subsection{Mantenimiento}

$\mathrm{Al}$ analizar el gráfico de la Figura 5, observamos que el bajo porcentaje de lascas de reactivación directa es acorde con el bajo número de artefactos tallados y el bajo nivel de talla de los mismos. A la vez, es un indicio de que el mantenimiento no fue una actividad de tiempo prolongado en los recintos. Observando los cuadros sobre el origen de las extracciones, el cuarzo es la única de las materias primas sobre la que encontramos lascas de reactivación directa: la preferencia de seguir trabajando sobre esta materia prima refleja la necesidad de aprovechar su tenacidad para una actividad específica.

Con respecto a la etapa de uso de los instrumentos, resulta útil conocer la cantidad de filos de cada instrumento para evaluar de esta manera el aprovechamiento de cada materia prima (Franco y Borrero 1996). La presencia de instrumentos con más de un tipo de filo, puntas o superficies activas complementarias permite reconocer conductas relacionadas con la maximización de la materia prima (Odell 1994).

El Cuadro 10 nos indica que, excluyendo los artefactos de talla sumaria y los filos naturales con rastros complementarios, por su carácter intrínseco de expeditividad, gran parte de los artefactos tallados sobre las materias primas locales no exhiben filos complementarios. Esto concuerda con un concepto de diseño utilitario (Escola 2000), en el que la motivación a la hora de producir dichos instrumentos se encontraba unida a una actividad específica y no era necesario aprovechar al máximo la materia prima.

En el mismo Cuadro 10 aparecen las mediciones respectivas al ángulo real del filo o ápice activo (Aschero 1975 y 1983) de los instrumentos. Esta medida ha sido 
utilizada como indicadora de su grado de mantenimiento. Al restar el valor del ángulo medido del valor del ángulo estimado -es decir, el ángulo correspondiente a las facetas de los lascados productos de la talla «recortados» por rastros complementarios o microlascados (Aschero 1975) - obtendremos a la par un indicador del desgaste del filo activo. Todos los instrumentos de andesita, rocas metamórficas y cuarzo conservaban sus filos activos (con un mínimo desgaste); por lo tanto, eran herramientas que participaban en la vida cotidiana de las sociedades formativas en el momento de ser abandonadas.

Cuadro 10: Indicadores de mantenimiento y desgaste de los artefactos tallados ( $\mathrm{N}=9)$

\begin{tabular}{lccccc}
\hline \multicolumn{1}{c}{ Grupos tipológicos } & $\begin{array}{c}\text { Ángulo } \\
\text { inicial }\end{array}$ & $\begin{array}{c}\text { Ángulo } \\
\text { medido }\end{array}$ & $\begin{array}{c}\text { Ángulo } \\
\text { desgaste }\end{array}$ & $\mathbf{N}^{\mathbf{0}}$ de filos & Reactivación \\
\hline Raspador & 48 & 33 & 15 & 1 & no \\
Cuchillo & 40 & 38 & 2 & 1 & no \\
Raspador & 78 & 58 & 20 & 1 & no \\
Raspador & 52 & 42 & 10 & 2 & no \\
Punta de proyectil & 54 & 52 & 2 & 1 & no \\
Punta entre muescas & 63 & 43 & 10 & 1 & sí \\
Punta de proyectil & 48 & 39 & 9 & 1 & sí \\
Punta de proyectil (C88 R2) & 48 & 36 & 12 & 1 & sí \\
Raedera (C88 R2) & 50 & 39 & 11 & 1 & no \\
\hline
\end{tabular}

En relación con esto último, ninguno de los artefactos tallados de los dos recintos cuenta con filos embotados o micro-astilladuras por el uso. En contraposición, observamos sobre los proyectiles de obsidiana actividades tendentes a prolongar la vida útil de estos artefactos. Se detectan acciones de reactivación a través de la superposición de retoques (observándose secuencias de lascados de talla más recientes). Creemos que la reactivación puede ser explicada a través de una decisión de economizar una materia prima que provenía de una región lejana. Por otra parte, se tratan de instrumentos afectos a una actividad como la caza, la cual habría adquirido relevancia ante épocas de sequías, con disminución de los rebaños cautivos.

\section{Discusión y conclusiones}

Consideramos que los resultados aquí presentados contribuyen a acrecentar el conocimiento sobre la relación que mantenían las sociedades agro-pastoriles plenas con sus recursos líticos.

Acerca de la funcionalidad de ambos recintos, Lanzelotti (2012) establece que para el caso de la Unidad C48 R3 se trataría de una estructura para almacenamiento o dormitorio, en virtud de la gran cantidad de fragmentos cerámicos que podrían haber sido utilizados como contenedores. El recinto C88 R2 es interpretado, para momentos tempranos, también como un dormitorio o lugar de almacenamiento, mientras que para los momentos tardíos es factible que fuera remodelado por quienes administraban la represa arqueológica (Lanzelotti 2012). Con la finalidad de colaborar en esta 
interpretación, a través de la información vertida anteriormente podemos sintetizar que en los dos recintos: a) se seleccionaron materias primas de diversa calidad; b) se explotaron en forma diferencial las materias primas de origen inmediatamente local; c) existe un desequilibrio entre el número de instrumentos y el conjunto lítico total; d) el esfuerzo tecnológico registrado en los instrumentos es bajo, con la excepción de las puntas de proyectil; e) existen evidencias de la persistencia de actividades predadoras como la caza entre las comunidades formativas y las del Período Tardío; f) las etapas de producción que predominan en las dos muestras son las de talla y regularización de filos.

Existen algunas características de la tecnología lítica analizada que sugerimos que es necesario destacar, en gran medida como líneas de investigación en un futuro cercano.

En primer lugar, el aprovechamiento de materias primas como el cuarzo, que a pesar de su tenacidad que dificulta la talla, aparece en una elevada frecuencia en el conjunto lítico, tanto del sitio que nos ocupa y como en zonas cercanas, como Horco Molle (Míguez et. al 2009), Quebrada de Los Corrales (Oliszewski et.al 2010) y particularmente en el Poblado Tardío de Rincón Chico (Gaál 2011). A partir del bajo grado de talla de los instrumentos, es dable pensar que tanto las comunidades formativas como las sociedades del Tardío utilizaban las materias primas locales con un fin específico e inmediato, que fueran útiles para una actividad concreta, para luego ser desechados sin necesidad de mantenimiento alguno, lo cual nos indica una continuidad en una decisión tecnológica a través del tiempo. No obstante, sugerimos indagar si en realidad, en el caso concreto del cuarzo, la tenacidad no fue acaso una propiedad explícitamente requerida por los talladores. En otras palabras, es necesario ahondar en el conocimiento que poseían estas comunidades de Caspinchango, ya que tanto en sus prácticas de talla como en la búsqueda de materias primas ponían en juego un saber. Este no pertenecía al sujeto en sí mismo, sino que había sido socializado, dado que el conocimiento es un fenómeno intersubjetivo.

En segundo lugar, sugerimos que existió en el Valle de Yocavil una fuerte asociación entre las obsidianas (como recurso lítico) y la confección de las puntas de proyectil, especialmente si sumamos a los datos registrados aquí los de otros sitios del valle, como el sitio formativo Soria 2 (Carbonelli 2011) y el poblado tardío de Rincón Chico (Gaál 2011). Debemos considerar que, además de las características intrínsecas de la obsidiana, que por su estructura vítrea cuenta con la capacidad de dañar efectivamente a las presas, pudieron existir factores sociales con intervención en la forma de organizar la producción. Diversos autores (Escola 2007; Lazzari 2008) han planteado la inclusión de dicha materia prima en una red de intercambio, que no estaría estrictamente ligada a actividades de subsistencia, sino a una necesidad de reproducción social de las sociedades formativas, que habrían requerido de contactos y lazos personales con otras regiones del Noroeste Argentino. A esto debemos agregar la existencia, en el Valle de Yocavil, de una continuidad temporal en el consumo de las mismas variedades de obsidianas: para el Formativo encontramos presencia de las fuentes Laguna Cavi y Ona-Las Cuevas en Soria 2 (Carbonelli 2011) y de Ona-Las Cuevas en el recinto C48 de Caspinchango, reproduciéndose este modelo en el Tardío, ya que se ha registrado la presencia de obsidianas de la fuente Ona-Las Cuevas 
en el poblado de Rincón Chico (Yacobaccio et al. 2004) y, como vimos aquí, de la fuente Laguna Cavi en el recinto C88 de Caspinchango.

Finalmente, y en tercer lugar, con respecto al proceso de manufactura en sí, el sitio Mesada del Agua Salada coincide con las tendencias generales marcadas por Hocsman (2006) y Hocsman y Escola (2006-07) para la región de Antofagasta de la Sierra. A fin de evaluar, a través del tiempo, el grado de inversión de energía y tiempo en los procesos de talla, Hocsman (2006) segmentó la información arqueológica de Antofagasta de la Sierra en tres grupos cronológicos: 5900-4350 años AP, 4260-3200 años AP, 2180-1340 años AP. Por la distribución de los conjuntos habitacionales, la presencia de estilos cerámicos recuperados en excavación y los fechados obtenidos (Lanzelotti 2012), los dos recintos del sitio Mesada del Agua Salada se integrarían en el tercer grupo cronológico. Los datos recolectados de los sitios puneños indican que, a medida que nos acercamos al Grupo Cronológico 3, el adelgazamiento bifacial y unifacial y la reducción unifacial desaparecen; se incrementan el uso de la técnica de reducción bifacial (orientado a la confección de puntas de proyectil) y el trabajo no invasivo bifacial. Sin embargo, la mayor frecuencia de técnicas de talla registrada en estos grupos corresponde al trabajo no invasivo unifacial (Hocsman 2006; Hocsman y Escola 2006-07). En los análisis tecnológicos realizados sobre los artefactos tallados de los dos recintos de Mesada del Agua Salada, registramos 14 artefactos con trabajo no invasivo unifacial y 2 artefactos con reducción bifacial, precisamente las puntas de proyectil de obsidiana. De esta manera, los resultados de tales análisis constituyen una de las primeras vías exploratorias para afirmar que en contextos agropastoriles plenos, tanto en Puna como en Valles, existió la convergencia de tres opciones tecnológicas: la reducción bifacial, el trabajo no invasivo bifacial y el unifacial (Hocsman y Escola 2006-07).

\section{Referencias bibliográficas}

Álvarez, Myriam

2004 «Producción lítica en el pukará de Tilcara». Mosaico. Trabajos en Antropología social y Arqueología 1: 25-33.

ANDREFSKY, William

1998 Lithics. Macroscopic approaches to analysis. Cambridge: Cambridge University Press.

Aragón, Eugenio y Nora V. Franco

1997 «Características de rocas para la talla por percusión y propiedades petrográficas». Anales del Instituto de la Patagonia (Ser. Cs. Hs) 25: 187-199.

Aschero, Carlos

1975 «Ensayo para una clasificación morfológica de artefactos líticos aplicados a estudios tipológicos comparativos». Informe inédito presentado al CONICET. Buenos Aires.

1983 «Revisiones. Ensayo para una clasificación morfológica de artefactos líticos. Apéndices A y B». Manuscrito inédito. Cátedra de Ergología y Tecnología, Universidad de Buenos Aires. 
Aschero, Carlos y Salomón Hocsman

2004 «Revisando cuestiones tipológicas en torno a la clasificación de artefactos bifaciales», en Temas de arqueología, análisis lítico, A. Acosta, D. Loponte y M. Ramos, eds., pp.7-25. Luján: Universidad Nacional de Luján.

Aschero, Carlos, Laura Moya, Claudia Sotelos y Jorge Martínez

1995 «Producción lítica en los límites del bosque cordillerano: el sitio Campo Río Roble 1». Relaciones de la Sociedad Argentina de Antropología 20: 205-238.

Avalos, Julio

2003 «Sistemas de producción lítica de las sociedades tardías de la Quebrada de Humahuaca». Cuadernos de la Facultad de Humanidades y Ciencias Sociales de Jujuy 20: 271-290.

Bellelli, Cristina

1991 «Los desechos de talla en la interpretación arqueológica. Un sitio de superficie en el Valle de Piedra Parada (Chubut)». Shincal 3: 79-93.

Bellelli, Cristina y Débora KLIGManN

1996 «Identificación de procesos de producción lítica a través del análisis de los desechos de talla», en Arqueología: sólo Patagonia. II Jornadas de Arqueología de la Patagonia, J. Gómez Otero, ed., pp. 307-317. Puerto Madryn: Centro Nacional Patagónico.

Carbonelli, Juan Pablo

2009a Interacciones cotidianas entre materias primas y sujetos sociales en el Valle de Yocavil. El caso del sitio Soria 2 (Andalhuala, Pcia. de Catamarca). Tesis de licenciatura. Buenos Aires: Universidad de Buenos Aires.

2009 b «La tecnología lítica desde las sociedades recolectoras a las sociedades formativas en el Valle de Yocavil (Catamarca)». Comenchingonia Virtual 3 (1): 95-113.

2011 «'Motivos porque y para' en la tecnología lítica de un sitio formativo en el Valle de Yocavil». Intersecciones en Antropología 12: 31-44.

CARdillo, Marcelo y Victoria Nuviala

2004 «Análisis de la diversidad y distribución de núcleos en el área costera de San Julián. Implicancia para el estudio de las estrategias de producción y uso de los artefactos líticos». Trabajo presentado en la I Jornada de Jóvenes Investigadores en Ciencias Humanas. Bahia Blanca.

Chaparro, María Gabriela

2002 «Informe de análisis lítico del sitio Tolombón, Provincia de Salta. Trabajo de campo año 2001». Intersecciones en Antropología 3: 119-123.

2007 «Preferencias en el manejo cotidiano de rocas. Los artefactos líticos de los asentamientos estatales del sur de la Quebrada de Humahuaca y el valle Calchaquí medio (Argentina)», en Al borde del Imperio. Paisajes sociales en áreas periféricas del Qollasuyu, V. Williams y B. Cremonte, eds. En prensa.

2008-09 «La tecnología en Tolombón: nuevas contribuciones al estudio de las Sociedades Tardías del NOA». Anales de Arqueología y Etnología 64-65: 107-136.

2009 El manejo de los recursos líticos en el pasado: sociedades pre-estatales y estatales en el área valliserrana del Noroeste Argentino (1000-1536). Tesis doctoral. Buenos Aires: Área de Arqueología, Universidad de Buenos Aires.

Cigliano, Eduardo

1960 Investigaciones arqueológicas en el Valle de Santa María. Rosario: Publicación del Instituto de Antropología, Universidad Nacional del Litoral. 
Civalero, María Teresa y Nora V. Franco

2003 «Early Human Occupations at the West of Santa Cruz Province, Southernmost South America». Quaternary International 109-110: 77-86.

Debenedetti, Salvador

1921 «La influencia hispánica en los yacimientos arqueológicos de Caspinchango (provincia de Catamarca)». Publicaciones de la Sección Antropológica 20: 745-788.

ELÍAs, Alejandra M.

2007 «Tecnología lítica en las sociedades tardías de Antofagasta de la Sierra (Puna Meridional Argentina)». Estudios Atacameños 33: 59-85.

2010 Estrategias tecnológicas y variabilidad de los conjuntos líticos de las sociedades tardias en Antofagasta de la Sierra (Provincia de Catamarca, Puna Meridional Argentina). Tesis doctoral. Buenos Aires: Área de Arqueología, Universidad de Buenos Aires.

ELÍAs, Alejandra M. y Patricia S. Escola

2010 «Viejos y nuevos horizontes: obsidianas entre las sociedades agrícolas-pastoriles del Período Tardío en Antofagasta de la Sierra (provincia de Catamarca, Puna Meridional Argentina)». Revista Española de Antropología Americana 40 (2): 29-49.

ERICSON, Jonathon E.

1984 «Toward the Analysis of Lithic Production Systems. Prehistoric Quarries and Lithic production», en New Directions in Archaeology, J. E. Ericson y B. A. Purdy, eds., pp.1-10. Cambridge: Cambridge University Press.

Escola, Patricia S.

1987 Las puntas de proyectil del Formativo en Puna y Quebradas de acceso: un estudio tecno-tipológico de cuatro casos de análisis. Tesis de licenciatura. Buenos Aires: Universidad de Buenos Aires.

2000 Tecnología Lítica y sociedades agropastoriles tempranas. Tesis doctoral. Buenos Aires: Universidad de Buenos Aires.

2007 «Obsidianas en contexto: tráfico de bienes, lazos sociales y algo más», en Sociedades precolombinas surandinas, Verónica I. Williams, Beatriz N. Ventura, Adriana Callegari y Hugo D. Yacobaccio, eds, pp. 73-87. Buenos Aires.

Escola, Patricia S. y Salomón Hocsman

2007 «Procedencia de artefactos de obsidiana de contextos arqueológicos de Antofagasta de la Sierra (ca. 4500-3500 AP)». Comechingonia 10: 49-61.

EsPINOSA, Silvana

1995 «Dr. Scholl y Monsieur Fleur: De talones y bulbos». Cuadernos del Instituto Nacional de Antropología y Pensamiento Latinoamericano 16: 315-327.

1996 «Descubriendo desechos. Análisis de desechos de talla lítica»», en Arqueología: sólo Patagonia. II Jornadas de Arqueología de la Patagonia, J. Gómez Otero, ed., pp. 333-339. Puerto Madryn: Centro Nacional Patagónico.

Flores, Marina y Federico WynVeldT

2009 «Análisis tecno-tipológico de los artefactos líticos de la Loma de los Antiguos de Azampay (Departamento de Belén, Catamarca)». Intersecciones en Antropología 10: 221-235.

Franco, Nora V. y Luis A. Borrero

1996 «El Stress Temporal y los Artefactos Líticos. La Cuenca Superior del Río Santa 
Cruz», en Arqueología Sólo Patagonia. II Jornadas de Arqueología de la Patagonia, J. Gómez Otero, ed., pp. 341-348. Puerto Madryn: Centro Nacional Patagónico.

GAÁL, Erico G.

2011 «Un acercamiento preliminar al estudio de la base local de recursos líticos y al análisis artefactual en el Poblado Arqueológico de Rincón Chico (período Tardío), Valle de Yocavil, Catamarca». Comechingonia Virtual 5 (1): 1-38.

GaÁl, Erico y Juan Pablo CARBonelli

2009 «Caracterización de las materias primas líticas y la secuencia de producción del sitio formativo Soria 2, Andalhuala, Prov. de Catamarca». Trabajo presentado en las Octavas Jornadas de Jóvenes Investigadores en Ciencias Antropológicas. Buenos Aires.

GonzÁlez, Luis R y Myriam N. TARragó

2004 «Dominación, resistencia y tecnología: La ocupación incaica en el Noroeste Argentino». Chungará 36 (2): 391-404.

Hocsman, Salomón

2006 «Tecnología lítica en la transición de cazadores recolectores a sociedades agropastoriles en la porción meridional de los Andes Centro Sur». Estudios Atacameños 32: $59-73$.

Hocsman, Salomón y Patricia S. Escola

2006-07 «Inversión de trabajo y diseño en contextos líticos agro-pastoriles (Antofagasta de la Sierra, Catamarca)». Cuadernos del Instituto de Antropología y Pensamiento Latinoamericano 21: 75-90.

Hocsman, Salomón, Carolina Somonte, María del Pilar Baвot, Álvaro Rodrigo Martel y Andrea Toselli

2003 «Análisis de los materiales líticos de un sitio a cielo abierto del área valliserrana del NOA: Campo Blanco, Tucumán». Cuadernos de la Facultad de Humanidades y Ciencias Sociales 20: 325-350.

LANZELOTTI, Sonia

2012 Uso del Espacio y construcción del paisaje agrícola en la cuenca del río Caspinchango, Valle de Yocavil, provincia de Catamarca. Tesis doctoral. Buenos Aires: Área de Arqueología, Universidad de Buenos Aires.

Lanzelotti, Sonia L., Alina Álvarez Larrain, Marcelo Lamamí y Gabriel E. Acuña

2010 «La espacialidad en Caspinchango: primera aproximación a la distribución de conjuntos arquitectónicos en la «Mesada del Agua Salada». Trabajo presentado en el XVII Congreso Nacional de Arqueología Argentina. Mendoza.

LAZZARI, Marisa

1998 «La economía más allá de la subsistencia: intercambio y producción lítica en el Aconquija». Arqueología 7: 9-49. Buenos Aires.

1999 «Objetos viajeros e imágenes espaciales: las relaciones de intercambio y la producción del espacio social». Revista do Museo de Arqueologia e Etnologia 3: 371385. São Paulo.

LEDESMA, Rossana

2003 «Diseños de puntas de proyectil. Una vía de análisis alternativo para el estudio de la identidad en la Quebrada del Toro, Provincia de Salta, Argentina». Cuadernos de la Facultad de Humanidades y Ciencias Sociales de Jujuy 20: 241-263. 
Míguez, Gabriel, Jorge Funes Coronel y Carlos M. Gramajo BüHler

2009 «Tecnología lítica en el piedemonte tucumano durante el Formativo. El caso de Horco Molle». La Zaranda de Ideas 5: 133-147.

NAmi, Hugo y Cristina Bellelli

1994 «Hojas, experimentos y análisis de desechos de talla. Implicaciones arqueológicas para la Patagonia centro-septentrional». Cuadernos del Instituto Nacional de Antropología y Pensamiento Latinoaméricano 15: 199-223.

ODELl, Gregory

1994 «Assessing Hunter-Gatherer Mobility in the Illinois Valley: Exploring Ambiguous Results», en The Organization of North American Prehistoric Chipped Stone Tool Technologies, P. J. Carr, ed., pp. 70-86. Nueva York: Plenum Press.

OLIVERA, Daniel E.

1988 «La opción productiva: apuntes para el análisis de sistemas adaptativos de tipo Formativo del Noroeste Argentino», en Precirculados de las Ponencias Cientificas a los Simposios del IX Congreso Nacional de Arqueología Argentina, pp. 88101. Buenos Aires: Instituto de Ciencias Antropológicas, Universidad de Buenos Aires.

Oliszweski, Nurit, Guillermo Arreguez, Hernán Cruz, Eduardo Di Lullo, Carlos M. Gramajo Bühler, Eduardo P. Mauri, Martín M. Pantorrilla Rivas y María Gabriela Srur

2010 «Puesto Viejo: una aldea temprana en la Quebrada de los Corrales (E1 Infiernillo, Tucumán)», en Actas del XVII Congreso Nacional de Arqueología Argentina, pp. 1697-1702. Mendoza: Facultad de Filosofía y Letras, Universidad Nacional de Cuyo.

Scattolin, María Cristina, M. Fabiana Bugliani, Andrés D. Izeta, Marisa Lazzari, Lucas Pereyra Domingorena y Leticia Martínez

2001 «Conjuntos materiales en dimensión temporal. El sitio formativo «Bañado Viejo» (Valle de Santa María, Tucumán)». Relaciones de la Sociedad Argentina de Antropología 26: 167-192.

Somonte, Carolina

2005 «Uso del espacio y producción lítica en Amaicha del Valle (Departamento Tafí del Valle, Tucumán)». Intersecciones en Antropología 6: 43-58.

2009 Tecnología lítica en espacios persistentes de Amaicha del Valle (Tucumán). Tesis Doctoral. Buenos Aires: Universidad de Buenos Aires.

SPROVIERI, Marina

2007 «Producción lítica en las sociedades tardías del Valle Calchaquí (Salta)». Mundo de Antes 5: 91-118.

SPROVIERI, Marina y Lidia BALDINI

2007 «Aproximación a la producción lítica en sociedades tardías. El caso de Molinos I, Valle Calchaquí Central (Salta)». Intersecciones en Antropología 8: 135-147.

TARRAGÓ, Myriam N.

1987 «Sociedad y sistema de asentamiento en Yocavil». Cuadernos del Instituto Nacional de Antropología 12: 179-196.

1995 «Desarrollo social en Yocavil: una estrategia de investigación». Hombre y Desierto 9: 225-235.

1999 «El Formativo y el surgimiento de la complejidad social en el Noroeste argenti- 
no», en Formativo sudamericano: una reevaluación, P. Ledergerber-Crespo, ed., pp. 302-313. Quito: Abya-Yala.

VALVERDE, Federico

2003 «Análisis de los desechos líticos de la ocupación inicial del sitio cueva Tixi (Provincia de Buenos Aires): cadena operativa de producción y técnicas de talla tempranas». Relaciones de la Sociedad Argentina de Antropología 28: 185-202.

Yacobaccio, Hugo D., Patricia S. Escola, Fernando X. Pereyra, Marisa Lazzari y Michael D. GLASCOCK

2004 «Quest for Ancient Routes: Obsidian Sourcing Research in Northwestern Argentina». Journal of Archaeological Science 31 (2): 193-204. 